\title{
Presepsin Level Correlates with the Development of Moderate Coronary Artery Calcifications in Hemodialysis Patients: A Preliminary Cross-Section Design Study
}

This article was published in the following Dove Press journal:

Risk Management and Healthcare Policy

\author{
Ahmed F Elhabashi \\ Leena Sulaibeekh (D) \\ Nahed Seddiq \\ Salman Alali \\ Amjad K Abdulmajeed \\ Nuria S Perez
}

Bahrain Defense Force Hospital, Royal Medical Services, Riffa, Kingdom of

Bahrain
Correspondence: Ahmed F Elhabashi Bahrain Defense Force Hospital, Royal Medical Services, Riffa, Kingdom of Bahrain

Email afame73@gmail.com

\begin{abstract}
Purpose: End-stage renal disease patients have a high mortality rate linked to cardiovascular complications, and one of these complications is vascular calcification. This study was performed to test if presepsin, an inflammatory marker, is a predictor of coronary artery calcification (CAC) in hemodialysis (HD) patients.

Patients and Methods: This study was a cross-sectional design involving $48 \mathrm{HD}$ patients and 13 control subjects. Coronary artery calcification score (CACs) was evaluated by a high resolution, ECG synchronized computed tomography of the heart using a CT calcium scoring. Presepsin and other laboratory analyses were performed on blood samples drawn before HD.

Results: Presepsin levels in HD patients were 14 times higher than healthy controls $(\mathrm{P}<0.01)$. Also, all laboratory tests except for vitamin $\mathrm{D}$ were significantly different than controls. Presepsin, phosphorus levels, and calcium-phosphate product were positively correlated with increasing CACs within groups of zero to moderate calcifications $(p<0.05$, $\mathrm{R}=0.459$ and $<0.01, \mathrm{R}=0.591$, respectively). These correlations were not seen with eGFR, PTH, calcium, vitamin D, CRP, or ESR levels. Furthermore, the log-transformed data of presepsin correlated with $1-15$ months of HD vintage $(\mathrm{p}<0.05, \mathrm{R}=0.482)$, whereas CACs data correlated with $1-20$ months of $H D$ vintage $(\mathrm{p}<0.05, \mathrm{R}=0.425)$.

Conclusion: Although this study is preliminary and has a limited number of patients, it shows that presepsin, as an inflammatory marker, correlates with the development of moderate CAC in HD patients and may predict CAC development. Therefore, measuring presepsin and managing inflammation before and during the early phases of HD may lower coronary calcification development. However, more clinical studies in this direction are essential.
\end{abstract}

Keywords: presepsin, hemodialysis, end-stage renal disease, coronary arterial calcification

\section{Introduction}

It has been estimated that 2 million people worldwide suffer from end-stage renal disease (ESRD), and the rate of new cases per year reaches $7 \% .^{1}$ Depending on either managing or treating ESRD, ie, dialysis vs kidney transplantation, mortality rates vary very much. For instance, one year of dialysis could result in a $20-25 \%$ mortality rate, and a 5 -year survival rate is approximately $35 \%$. In kidney transplantation, however, the mortality rate drops to $3 \%$ after five years. ${ }^{1}$

In dialysis patients, the elevated mortality rate is highly linked to cardiovascular complications. ${ }^{2-5}$ Of these complications is vascular calcification (VC). Although VC is 
one of the aging processes defined by calcium deposits in one of the major vascular beds (coronary, carotid, and aorta), it has been documented that it occurs in two different patterns. ${ }^{2-5}$ One of these patterns occurs in the intimal layer and is associated with inflammation-induced atherosclerosis. ${ }^{6}$ While the other occurs in the medial layer and associated with chronic kidney disease (CKD) ${ }^{6,7}$ Previous studies have suggested that the $\mathrm{VC}$ in patients with CKD is not an inflammatory atherosclerotic calcification, but related to calcium and phosphate effects on vascular smooth muscle apoptosis and the production of calcifying matrix and extracellular matrix degradation. ${ }^{6,7}$ However, other reports have shown the majority of VC in CKD patients and patients on dialysis is of an inflammatory pattern. ${ }^{8-10}$ For instance, it has been shown that in patients with early as well as advanced CKD, the proinflammatory molecules are upregulated in the vascular wall and calcification of the aortic media compared to coronary heart patients with no non-renal involvement. Since VC is an active process involving the disorders of mineral and bone metabolism disorder, inflammation, oxidative stress, and their interactions, ${ }^{11}$ it would be of high interest to predict $\mathrm{VC}$ in CKD patients on hemodialysis (HD).

Presepsin is a $13 \mathrm{KD}$ N-terminal fragment of the cluster of differentiation (CD)-14. CD14 is $55 \mathrm{KD}$ receptor on the cell membrane of monocytes, macrophages, and neutrophils that binds to lipopolysaccharide (LPS) and LPS-binding protein complexes. ${ }^{12}$ Once binding occurs, intracellular and downstream pathways are evident, and one of the outcomes of these pathways is the release of presepsin. ${ }^{12,13}$ Therefore, high plasma presepsin level was thought to be related to sepsis. It has been found that measuring presepsin would be a biomarker of early sepsis and determination of sepsis severity. ${ }^{12-14}$ Furthermore, high levels of plasma presepsin concentrations were also associated with an increased incidence of cardiovascular disease and all-cause mortality in the elderly, suggesting that presepsin could be a marker of inflammatory cardiovascular dysfunction. ${ }^{15}$

Since presepsin is $13 \mathrm{KD}$, it should be filtered by the glomerulus, reabsorbed, and catabolized within proximal tubular cells. ${ }^{16}$ However, in patients with CKD, it has been found the plasma presepsin level is high, and it is negatively correlated with low glomerular filtration rate (GFR). ${ }^{17}$ Furthermore, it has been found the plasma presepsin level is even higher in HD than in non-HD CKD patients. ${ }^{17}$ Although it has been reported that $\mathrm{VC}$ in patients with CKD is not an inflammatory atherosclerotic pattern, ${ }^{6,7}$ having high presepsin levels in the plasma in such patients and even higher in HD patients, raises the following questions. Why is the presepsin level extremely high in hemodialysis patients? Can this high presepsin level indicate inflammation in HD patients? Will this high presepsin level predict VC? Therefore, the present cross-sectional study was designed to evaluate presepsin levels in HD patients with different categories of coronary calcification (CAC).

\section{Patients and Methods Study Approval and Design}

A cross-sectional design protocol was submitted and approved by the Research Ethics Committee at the Royal Services of Bahrain Defense Force (BDF) Military Hospital (Approval \# BDF/R\&REC/2020-411) in early 2020. This study was carried out in accordance with the Declaration of Helsinki, where following the study approval, all subjects have read and signed an informed consent to enroll in the study.

\section{Patients and Control Subjects}

Forty-eight patients (38 males and 10 females), who had an ESRD and had been on HD for at least a month period were enrolled in the study. The HD is performed three times per week using hemodiafiltration modality with high flux filters. All supplement medications of calcium, calcimimetics, and vitamin D were given according to regular blood results for calcium, phosphate, PTH and vitamin D. Additionally, patients were evaluated for calcified coronary lesions by a high resolution, ECG synchronized computed tomography of the heart using a CT calcium scoring (syngo.CT CaScoring VB30). None of the patients experienced any kind of infection for several weeks before enrolling in the study. As for the control subjects $(n=13)$, they have been selected based on normal kidney functions, not having any kind of infection, and were included for the evaluation of presepsin and other biochemical tests.

\section{Presepsin and Other Biochemical Tests}

Blood was withdrawn from all participants before undergoing HD. Plasma and serum samples were obtained from each blood sample for performing biochemical tests, whereas EDTA-whole blood was kept for hematological tests. Plasma presepsin concentrations were measured using an automated chemiluminescent enzyme immunoassay technology and Magtration ${ }^{\circledR}$ technology $\left(\right.$ Pastfast $^{\mathrm{TM}}$, LSI Medience Corp. Tokyo, Japan). The measurement range of presepsin assay is $20-20,000 \mathrm{pg} / \mathrm{mL}$. In addition, 
other laboratory tests such as high-sensitive C-reactive protein (CRP), calcium, phosphorus (Cobas 6000, Roche), parathyroid hormone (PTH) (Cobas 8000, Roche), vitamin $\mathrm{D}$, and erythrocyte sedimentation rate (ESR) (Alifax, Padova, Italy; accelerated method) were performed. The kidney residual function was evaluated by estimating GFR levels (eGFR) prior to initiation of dialysis according to the modification of diet in renal disease equation.

\section{Statistical Analysis}

Continuous data are expressed depending on the skewness score of $>2$ or $<-2$ as either the means \pm SD or median (interquartile range), whereas categorical data are expressed as a percentage. The Student's $t$-test was applied for testing between two groups when both $\mathrm{n} \geq 8$ and skewness score was less than 2. For Student's $t$-test, the equal variance was assumed unless Levene's test was significant. For groups with smaller n, Mann-Whitney was applied. Similarly, ANOVA for means or the non-parametric Kruskal-Wallis test for medians was used to compare the quantitative variables among groups, when appropriate. For correlation studies, data variables either analyzed by non-parametric Spearman correlation or transformed to log transformation followed by performing data linear regression analysis when appropriate. The univariate and multivariate analysis for log presepsin and CACs were performed against multiple categorized factors. Finally, the frequency of conditions was tested using chi-square analysis. P-value of $<0.05$ was considered significant.

\section{Results}

\section{Characteristics and Clinical results of the Studied Subjects}

We included $48 \mathrm{HD}$ patients and 13 controls. Control subjects were not age or sex-matched with patients, but they were included to compare the clinical laboratory results with the patients' results.

Presepsin levels in HD patients were 14 times higher than control subjects $(\mathrm{p}<0.01)$. Furthermore, and as expected, the clinical laboratory results such as calcium, phosphorus, PTH, and CRP levels were significantly different in HD patients than control subjects (Table 1). Although significantly different, serum calcium and phosphorus levels in HD patients were within the reference ranges $(8.6-10.0 \mathrm{mg} / \mathrm{dl}$ and $2.5-4.5 \mathrm{mg} / \mathrm{dl}$, respectively) and this is due to the regulatory
Table I Characteristics of the Subjects Under Study

\begin{tabular}{|l|l|l|l|}
\hline Parameters & ESRD (n=48) & Control $(\mathbf{n}=13)$ & $P$ \\
\hline Age & $58.1 \pm 12.5$ & $38.4 \pm 12.0$ & $<0.001$ \\
Sex (M\%) & $79.2 \%$ & $15.3 \%$ & $<0.001$ \\
Diabetes & $81 \%$ & 0 & $<0.001$ \\
Hypertension & $96 \%$ & $15 \%$ & $<0.001$ \\
Dyslipidemia & $90 \%$ & $85 \%$ & NS \\
eGFR & $5.15(2.25)$ & $>60$ & $<0.001$ \\
Calcium (mg/dl) & $8.8 \pm 0.7$ & $9.6 \pm 0.3$ & $<0.001$ \\
Phosphorus (mg/dl) & $4.8 \pm 1.5$ & $3.5 \pm 0.5$ & $<0.001$ \\
Ca.Phos Products $\left(\mathrm{mg}^{2} / \mathrm{dl}^{2}\right)$ & $42.2 \pm 13.5$ & $33.6 \pm 4.9$ & $<0.001$ \\
PTH (pg/mL) & $210(225)$ & $27(18)$ & $<0.001$ \\
Vitamin D (ng/mL) & $19.1(10.5)$ & $21.6(19.4)$ & NS \\
CRP (mg/l) & $7.4(16.5)$ & $1.2(4.1)$ & $<0.01$ \\
ESR (mm/h) & $49(36)$ & $26(21)$ & $<0.01$ \\
Presepsin (ng/mL) & $1531(1347)$ & $111(62)$ & $<0.01$ \\
\hline
\end{tabular}

Note: Data are expressed as mean means $\pm S D$ or median (interquartile range), whereas categorical data are expressed as percentage.

response of high PTH levels (median $210 \mathrm{pg} / \mathrm{mL}$; Reference Range $16-65 \mathrm{pg} / \mathrm{mL}$ ) (Table 1).

\section{Classification of ESRD Patients: Presepsin Levels in HD Patients Correlate with Minimal to Moderate CACs}

ESRD patients were classified according to their calcification scores CACs (0->1000) or CA-CT (1-6); 0 to minimal (1-2 or $0-10)$, mild (3 or 11-100), moderate (4 or 101-400), significant (5 or 401-1000), and highly significant (6 or $>1000)$ (Table 2). ${ }^{18}$ Furthermore, most of the ESRD patients have hypertension $(95.6 \%)$, diabetes $(85.4 \%)$, or dyslipidemia (89.6\%), whereby $77.1 \%$ of patients has three conditions combined.

None of the biochemical analyses, such as eGFR, calcium, phosphorus, PTH, vitamin D, CRP, ESR, and presepsin levels, were significantly different between the categorized calcification groups (Table 2). However, presepsin, phosphorus, and calcium phosphate (Ca-P) product levels were positively correlated with CACs groups of 0 to 400 ( $p<0.05$; $\mathrm{R}=0.459$, and $\mathrm{p}<0.01 ; \mathrm{R}=0.591, \mathrm{R}=0.565$, respectively) (Figure 1A-D). On the other hand, no correlations were observed between CACs and eGFR, vitamin D, PTH, calcium, CRP, or ESR levels.

In HD patients with no to minimal CACs (0-10 CACs score), presepsin levels were significantly higher (8 times higher), and calcium levels were significantly less than the control subjects $(p<0.01)$. On the other hand, phosphorus, CA-P, vitamin D, CRP, and ESR values were not significantly different than control subjects. 
Table 2 Characteristics of ESRD Patients According to Calcification Score

\begin{tabular}{|c|c|c|c|c|c|c|}
\hline \multirow[t]{4}{*}{ Parameters } & \multicolumn{5}{|c|}{ Calcification Score CA-CT } & \multirow[t]{4}{*}{$\mathbf{P}$} \\
\hline & $\mathbf{I - 2}$ & 3 & 4 & 5 & 6 & \\
\hline & \multicolumn{5}{|l|}{ CACs } & \\
\hline & $0-10$ & $11-100$ & $101-400$ & $401-1000$ & $>1000$ & \\
\hline Patients & $10.4 \%$ & $16.7 \%$ & $12.5 \%$ & $31.3 \%$ & $29.2 \%$ & \\
\hline Age & $52 \pm 13$ & $60 \pm 20$ & $58 \pm 10$ & $58 \pm 9$ & $59 \pm 13$ & NS \\
\hline Dyslipidemia & $80 \%$ & $75 \%$ & $100 \%$ & $81.3 \%$ & $100 \%$ & NS \\
\hline Hypertension & $80 \%$ & $100 \%$ & $83.3 \%$ & $100 \%$ & $100 \%$ & NS \\
\hline Diabetes & $60 \%$ & $87.5 \%$ & $100 \%$ & $80 \%$ & $78.6 \%$ & NS \\
\hline eGFR $\left(\mathrm{mL} / \mathrm{min} / \mathrm{l} .73 \mathrm{~m}^{2}\right)$ & $5.4(2.6)$ & $5.3(3.0)$ & $5.3(1.6)$ & $4.9(2.6)$ & $5.6(3.0)$ & NS \\
\hline Calcium (mg/dl) & $8.6 \pm 0.8$ & $8.8 \pm 0.8$ & $8.7 \pm 0.6$ & $8.7 \pm 0.8$ & $8.8 \pm 0.6$ & NS \\
\hline Phosphorus (mg/dl) & $3.4 \pm 0.9$ & $4.8 \pm 0.6$ & $5.7 \pm 0.6$ & $4.7 \pm 0.6$ & $5.0 \pm 0.6$ & NS \\
\hline Ca.Phos Products $\left(\mathrm{mg}^{2} / \mathrm{dl}^{2}\right)$ & $29.2 \pm 6.9$ & $42.2 \pm 13.4$ & $49.6 \pm 16.8$ & $40.9 \pm 14.0$ & $44 \pm 1.2 .9$ & NS \\
\hline PTH (pg/mL) & $320 \pm 111$ & $295 \pm 234$ & $191 \pm 132$ & $289 \pm 215$ & $233 \pm 137$ & NS \\
\hline Vitamin D (ng/mL) & $16.2(12.7)$ & $19.7(3.3)$ & I $4.6(4.8)$ & $20.2(12.0)$ & $21.2(12.9)$ & NS \\
\hline ESR $(\mathrm{mm} / \mathrm{h})$ & $49 \pm 20$ & $48 \pm 38$ & $61 \pm 20$ & $48 \pm 28$ & $60 \pm 26$ & NS \\
\hline CRP (mg/L) & $6.7(I 5.0)$ & $5.2(14.5)$ & $5.81(60.6)$ & $9.4(19.2)$ & $7.6(15.5)$ & NS \\
\hline Presepsin $(\mathrm{pg} / \mathrm{mL})$ & $909(964)$ & $1968(2842)$ & 1767 (5599) & $\mid 587(|22|)$ & $802(1165)$ & NS \\
\hline Dialysis vintage (months) & $7(26)$ & $33(4 I)$ & $14.5(7)$ & $38(242)$ & $28.5(36)$ & NS \\
\hline
\end{tabular}

Note: Data are expressed as mean means \pm SD or median (interquartile range), whereas categorical data are expressed as percentage.

\section{Increasing Presepsin is Associated with I-I5 Months HD Vintage}

The log-transformed CACs were significantly correlated with counterparts of HD vintage $(\mathrm{p}<0.01, \mathrm{R}=0.342)$. However, this was not observed between presepsin levels and $\mathrm{HD}$ vintage $(\mathrm{p}>0.05, \mathrm{R}=0.09)$ (Figure $2 \mathrm{~A}$ and $\mathrm{B})$. However, when HD vintage were categorized, logtransformed presepsin levels were significantly correlated with 1 to 15 months of HD vintage $(\mathrm{p}<0.05, \mathrm{R}=0.482)$, but not with CACs $(\mathrm{p}>0.05, \mathrm{R}=0.320)$ (Figure $2 \mathrm{C}$ and D). On the other hand, log CACs became significantly correlated with 1 to 20 months of HD vintage $(\mathrm{p}<0.05, \mathrm{R}=0.425)$.

Following categorizing the HD vintage, the presepsin level in group of 11-15 months of HD vintage was the highest $(p=0.071)$ and the CACs level in group of 16-30 months of HD was the highest $(\mathrm{P}<0.05)$.

\section{Presepsin Levels Were Correlated with CRP}

The log-transformed presepsin levels and CRP counterparts were highly correlated $(\mathrm{p}<0.01, \mathrm{R}=0.43$ ). On the other hand, no correlation between presepsin and eGFR, serum $\mathrm{Ca}++$, phosphorus, PTH or vitamin D was observed.

HD patients with hypertension, diabetes, and dyslipidemia have higher CACs but not presepsin.
Different combinations of hypertension, diabetes, and dyslipidemia and levels of presepsin and CACs were analyzed in ESRD patients. From our patients, only four different combinations were obtained. CACs, but not presepsin, were significantly higher in patients having hypertension, dyslipidemia, and diabetes than in patients who have none of the conditions $(\mathrm{p}<0.05)$ (Figure 3). However, no difference in CACs level was observed between other combinations, such as any two of the conditions versus having or not having the three conditions.

\section{Univariate and Multivariate Correlations of Presepsin with Other Risk Factors}

The univariate analysis of $\log$ presepsin data and the multivariate analysis of log presepsin and CACs with categorized factors such sex, age groups, hypertension, dyslipidemia, diabetes, HD vintage revealed no statistical significance correlation. However, when CACs of 0-400 patient's data were analyzed, the univariate analysis of $\log$ presepsin was significantly correlated with age groups $(\mathrm{p}<0.01)$, HD vintage $(\mathrm{p}<0.01)$ and CA-CT $(\mathrm{p}<0.05)$. Furthermore, the multivariate analysis of log presepsin and CACs showed significance with age groups and HD vintage $(\mathrm{p}<0.01)$.

\section{Discussion}

$\mathrm{VC}$ is a common outcome of CKD and mainly in ESRD. Therefore, predicting VC would help manage post ESRD 

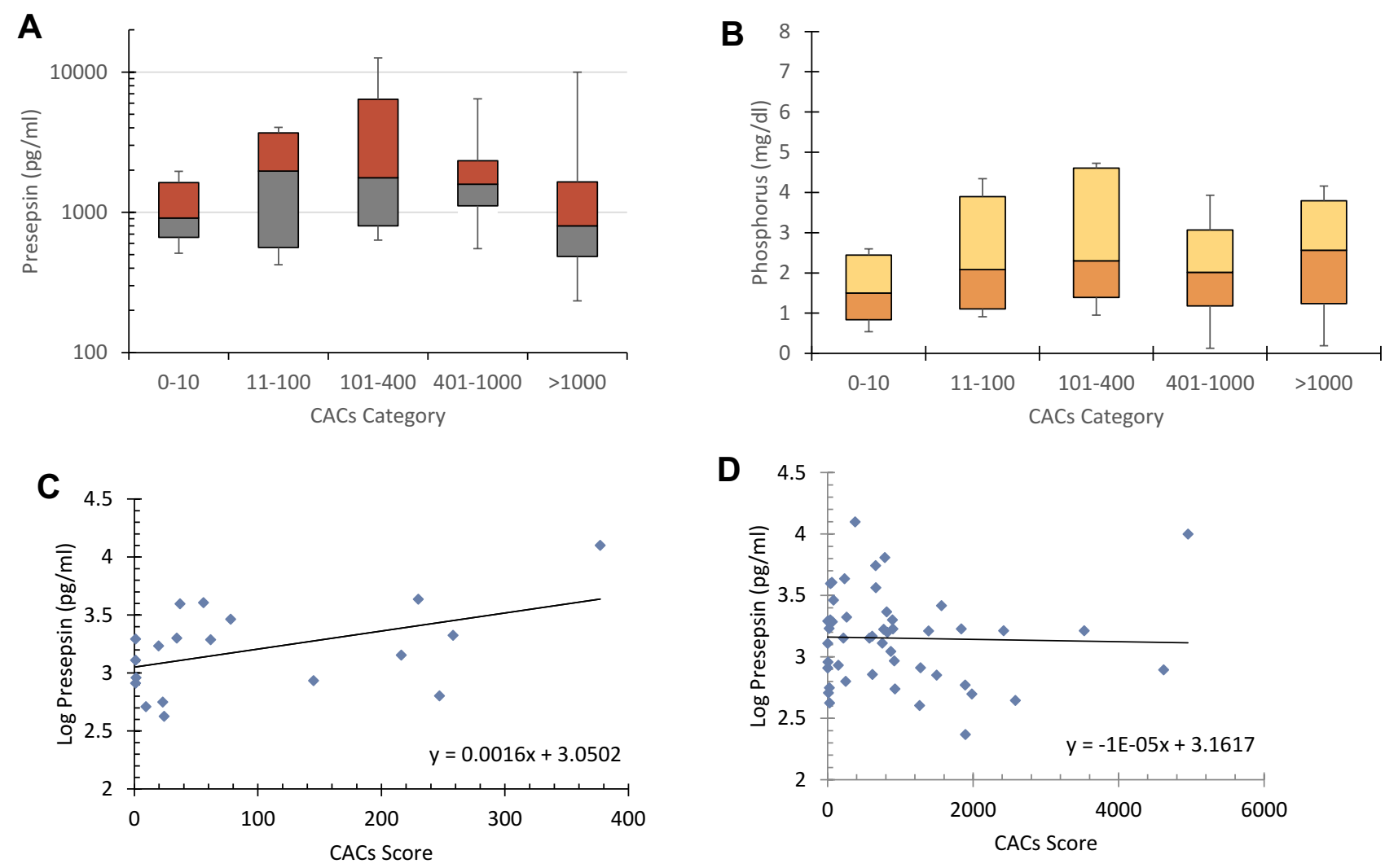

Figure I Box plot of presepsin (A) and serum phosphorus (B) in relation with CACs categories. Data shows an apparent increase in presepsin and phosphorus levels with increasing CACs up to $10 \mathrm{I}-400$ group in HD patients. (C) shows a significant correlation between $C A C s$ of $0-400$ and log presepsin levels ( $P<0.05$, $R=0.459$ ), whereas in (D) no correlation was observed when all CACs and log presepsin levels were included.

conditions before it happens. Since VC is predominantly an inflammatory condition, ${ }^{8-10}$ the search for an inflammatory marker to predict VC is highly essential.

Presepsin is one of the new inflammatory markers and been found to inversely correlated with GFR. ${ }^{17,19,20}$ Furthermore, in HD patients, presepsin levels are significantly higher than in grade 5 CKD patients. ${ }^{17}$ Similarly, in the present study, the overall presepsin level in HD patients was 14 times higher than renal-healthy controls. However, these high presepsin levels were not correlated with patients' low eGFRs levels $\left(5.5 \pm 2.4 \mathrm{~mL} / \mathrm{min} / 1.73 \mathrm{~m}^{2}\right)$.

Previous studies have suggested that the type of VC in patients with CKD is not an inflammatory atherosclerotic calcification but related to phosphorus and calcium disturbances. ${ }^{6,7}$ Our findings showed that plasma presepsin, and serum phosphorus, and calcium phosphate product levels were correlated with minimal to moderate CACs. Furthermore, serum phosphorus levels were significantly less in the minimal CACs than in the moderate CACs but not different than renal-healthy controls. In contrast, presepsin levels were $8 \mathrm{x}$ higher in HD patients who have minimal CACs than controls. These findings suggest that both inflammation and phosphorus disturbance in HD patients are related to the development of moderate CAC. ${ }^{8-11}$ However, the elevation of plasma presepsin proceeds that of phosphorus in CAC development indicating that systemic inflammation is more apparent and better predictor of $\mathrm{VC} .^{10}$ In CKD patients, microinflammation, but not Ca-P product, was evident in their vasculature tissues which implies that local microinflammation preceded Ca-P changes in the development of $\mathrm{VC}{ }^{10}$

Besides, presepsin levels were significantly correlated with 1-15 months of HD vintage. These findings might imply the importance of measuring presepsin in the early phases of HD, or even before the initiation of HD to follow up on CACs development. It has been established that chronic HD patients have immune dysfunction, mainly an imbalance between pro- and anti-inflammatory mechanisms. ${ }^{21-25}$ Also, it has been reported that a single session of HD could result in an immunological imbalance. $^{26}$ In dialysis patients, the VC of the inflammatory pattern was more prevalent than control subjects. ${ }^{9}$ In a cohort study, Nakayama et al (2011) reported that at the initiation of $\mathrm{HD}$, patients with carotid artery calcification 

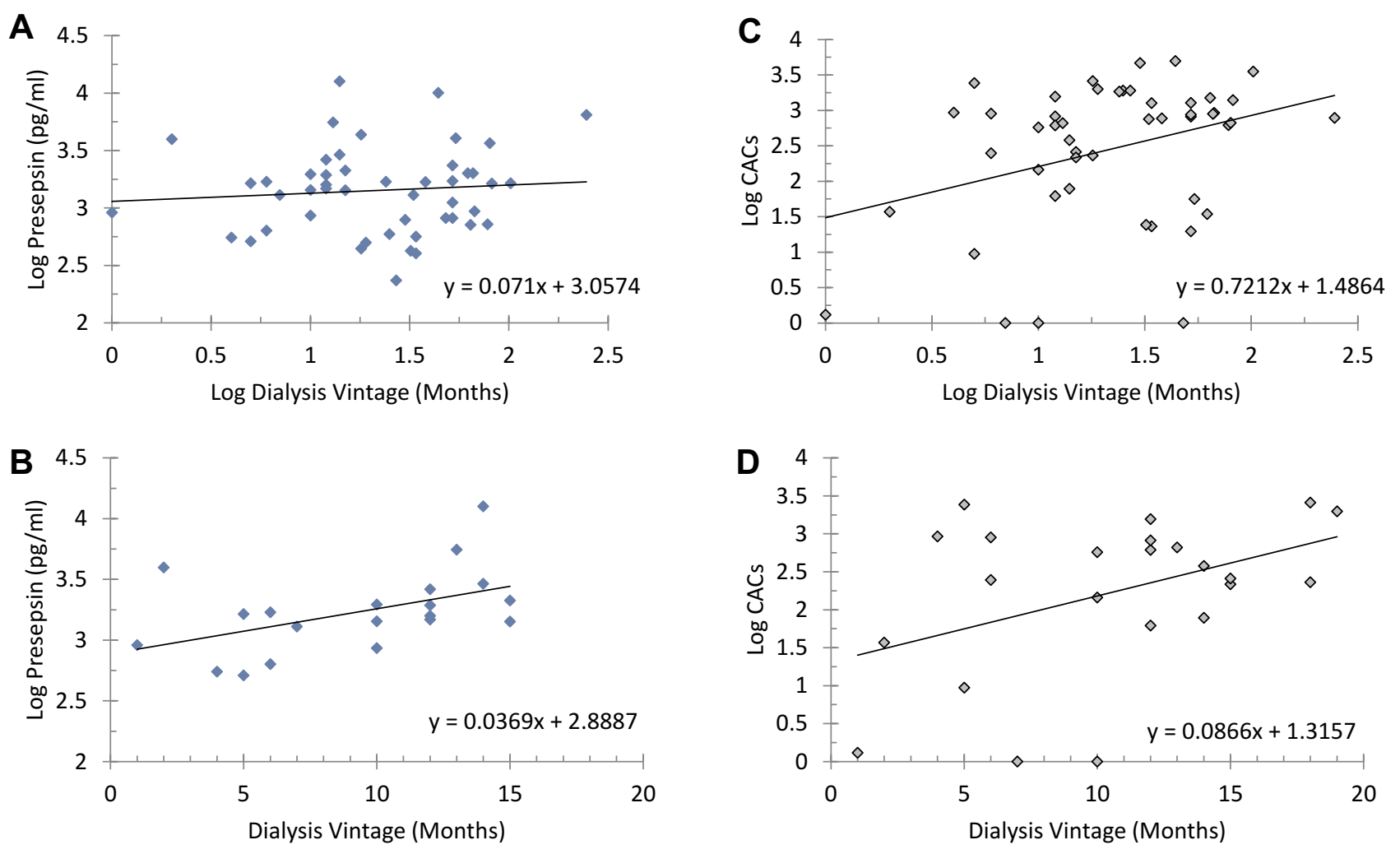

Figure 2 Correlation and linear regression analysis of log dialysis vintage and log CACs (A) or log presepsin levels (B) in HD patients is presented. A significant correlation between CACs and dialysis vintage was observed ( $p<0.05, R=0.342)$. When $H D$ vintage was categorized, a significant correlation between I-I5 months of $H D$ vintage and presepsin levels $(p<0.05, R=0.482)(C)$ and between $I-20$ months of HD vintage and CACs $(p<0.05, R=0.425)(D)$ were observed.

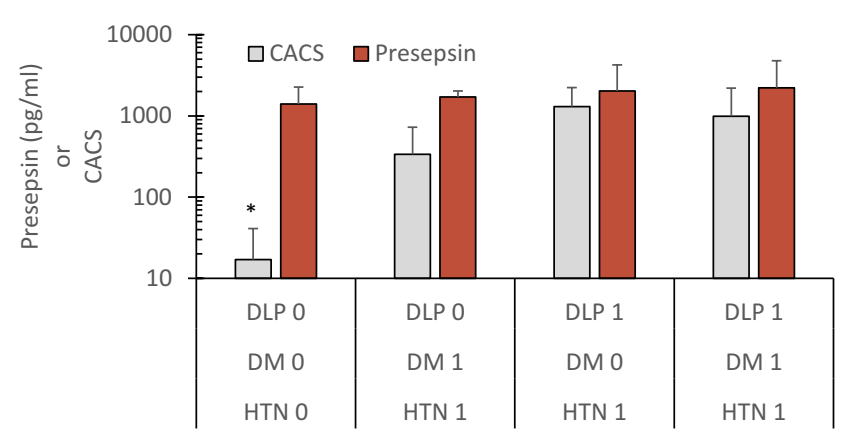

Figure 3 CACs and presepsin levels (mean \pm SD) in groups categorized according to hypertension (HTN), dyslipidemia (DLP), and diabetes mellitus (DP) positive/ negative conditions. CACs levels were significantly lower in patients with negative for HTN, DLP, and DM than patients having all the conditions combined $(* \mathrm{p}<0.05)$.

have a high probability of developing cardiovascular events than those with no calcification. ${ }^{20}$ The latter suggests that HD enhances cardiovascular events in patients with VC. However, these authors did not follow up on the incidence of carotid artery calcification. In a similar study, patients without CAC did not increase their CACs score over 18 months of HD, while those of moderate CACs significantly progressed to more severe calcification. ${ }^{27}$ In the present study, the HD vintage was correlated with presepsin levels, and then CACs followed. These findings suggest that HD vintage enhances systemic inflammation before CACs progression. This enhancement, however, may also be related to the existing inflammation just before the initiation of HD. ${ }^{18,28}$ It must be mentioned that mineral metabolism alteration plays an essential role in VC and also induces microinflammation. ${ }^{29}$ When HD is initiated, several calcification factors evolve. Of these vascular calcification inhibitors, such as fetuin-A and matrix Gla protein, are reduced in HD patients and have been inversely correlated with inflammation and VC. ${ }^{11,29}$ Additionally, it has been documented that in CKD patients and due to mineral and bone metabolism disorder, the high PTH plays a role on osteoblasts to induce the expansion and migration of circulating calcifying cells. ${ }^{11}$ These cells initiate signs of systemic inflammation before locating in a vasculature and induce $\mathrm{VC}$. $^{11}$

It has been reported that $30-50 \%$ of HD patients have elevated serum inflammatory markers such as CRP and interleukin-6 due to several reasons either related to the underlying cause of CKD or associated with the HD. ${ }^{22}$ In the present study, although CRP levels were elevated in 
HD patients, they were not correlated with CACs. On the other hand, presepsin levels were significantly associated with CRP levels, and increasing presepsin levels were correlated with mild to moderate CACs, suggesting that presepsin is a better indicator of CACs than CRP in minimal to moderate calcifications. CRP is an acute phase reactant produced from hepatocytes following an increase in proinflammatory cytokines. ${ }^{22,28}$ Presepsin, on the other hand, is a marker of activated monocytes/macrophages and probably of circulating calcifying cells of myeloid origin in CKD patients, ${ }^{11}$ therefore, higher presepsin would be a better inflammatory predictor in CAC development. However, why presepsin level plateaued or even dropped in significant and severe CACs is difficult to explain, but in such cases, $\mathrm{VC}$ becomes extremely unregulated, and multiple factors become involved. ${ }^{11,29}$

In this study, most patients ( $\sim 96 \%)$ have at least one of the three classic cardiovascular risk factors: hypertension, diabetes mellitus, and dyslipidemia, whereas $\sim 78 \%$ of the patients have the three conditions combined. Although undertreatment, HD patients with the three conditions have higher CACs, but not presepsin. ${ }^{5}$ Even though there are a small number of patients in two of the categorized groups, the accumulation of these three classic cardiovascular factors with systemic inflammation in place (already having high presepsin level) is associated with increasing CAC severity.

In conclusion, although this study is preliminary with a limited number of patients, it shows that presepsin as an inflammatory marker correlates with the development of moderate $\mathrm{CAC}$ in $\mathrm{HD}$ patients and may predict $\mathrm{CAC}$ development. Additionally, increasing presepsin level is associated with 1-15 months of HD vintage, and increasing CACs is associated with 1-20 months of HD vintage. Thus, it may be postulated that HD enhances preexisting inflammation, and this enhancement predicts VC. However, several studies with a larger patient sample directed towards measuring presepsin and managing inflammation before and during early phases of HD on CAC development are essential to validate this hypothesis.

\section{Disclosure}

The authors report no conflicts of interest for this work.

\section{References}

1. United States Renal Data System. 2018 USRDS annual data report: Epidemiology of kidney disease in the United States. National Institutes of Health, National Institute of Diabetes and Digestive and Kidney Diseases, Bethesda, MD, 2018.
2. Foley RN, Parfrey PS, Sarnak MJ. Clinical epidemiology of cardiovascular disease in chronic renal disease. Am J Kidney Dis. 1998;32 (Suppl 3):S112-S119. doi:10.1053/ajkd.1998.v32.pm9820470

3. London GM, Marchais SJ, Guérin AP, Métivier F. Arteriosclerosis, vascular calcifications and cardiovascular disease in uremia. Curr Opin Nephrol Hypertens. 2005;14:525-531. doi:10.1097/01.mnh. j0000168336.67499.c0

4. Blacher J, Guérin AP, Pannier B, Marchais SJ, London GM. Arterial calcifications, arterial stiffness, and cardiovascular risk in end-stage renal disease. Hypertension. 2001;38:938-942. doi:10.1161/ hy 1001.096358

5. Kobayashi S. Cardiovascular events in hemodialysis patients: challenging against vascular calcification. Ann Vasc Dis. 2017;10(1):1-7. doi:10.3400/avd.ra.17-00006

6. Hsu JJ, Lim J, Tintut Y, Demer LL. Cell-matrix mechanics and pattern formation in inflammatory cardiovascular calcification. Heart. 2016;102(21):1710-1715. doi:10.1136/heartjnl-2016-309667

7. Shanahan CM, Crouthamel MH, Kapustin A, et al. Arterial calcification in chronic kidney disease: key roles for calcium and phosphate. Circ Res. 2011;109:697-711. doi:10.1161/CIRCRESAHA.110. 234914

8. Nakamura S, Ishibashi-Ueda H, Niizuma S, Yoshihara F, Horio T, Kawano Y. Coronary calcification in patients with chronic kidney disease and coronary artery disease. Clin J Am Soc Nephrol. 2009;4:1892-1900. doi:10.2215/CJN.04320709

9. Coll B, Betriu A, Martínez-Alonso M, et al. Large artery calcification on dialysis patients is located in the intima and related to atherosclerosis. Clin J Am Soc Nephrol. 2011;6:303-310. doi:10.2215/CJN.04290510

10. Benz K, Hilgers KF, Daniel C, Amann K. Vascular calcification in chronic kidney disease: the role of inflammation. Int $J$ Nephrol. 2018;2018:4310379. doi:10.1155/2018/4310379

11. Cianciolo G, Capelli I, Cappuccilli M, Schillaci R, Cozzolino M, La Manna G. Calcifying circulating cells: an uncharted area in the setting of vascular calcification in CKD patients. Clin Kidney J. 2016;9:280-286. doi:10.1093/ckj/sfv145

12. Liu B, Chen YX, Yin Q, Zhao YZ, Li CS. Diagnostic value and prognostic evaluation of Presepsin for sepsis in an emergency department. Crit Care. 2013;17:R244. doi:10.1186/cc13070

13. Romualdo LG, Torrella PE, González MV, et al. Diagnostic accuracy of presepsin (soluble CD14 subtype) for prediction of bacteremia in patients with systemic inflammatory response syndrome in the emergency department. Clin Biochem. 2014;47:505-508. doi:10.1016/j. clinbiochem.2014.02.011

14. Ulla M, Pizzolato E, Lucchiari M, et al. Diagnostic and prognostic value of presepsin in the management of sepsis in the emergency department: a multicenter prospective study. Crit Care. 2013;17: R168. doi:10.1186/cc12847

15. Reiner AP, Lange EM, Jenny NS, et al. Soluble CD14. Genomewide association analysis and relationship to cardiovascular risk and mortality in older adults. Arterioscler Thromb Vasc Biol. 2013;33:158-164. doi:10.1161/ATVBAHA.112.300421

16. Chenevier-Gobeaux C, Trabattoni E, Roelens M, Borderie D, Claessens YE. Presepsin (sCD14-ST) in emergency department: the need for adapted threshold values? Clin Chim Acta. 2014;427:34-36. doi:10.1016/j.cca.2013.09.019

17. Nagata T, Yasuda Y, Ando M, et al. Clinical impact of kidney function on presepsin levels. PLoS One. 2015;10(6):e0129159. doi:10.1371/journal.pone.0129159

18. Rumberger JA, Brundage BH, Rader DJ, Kondos G. Electron beam computed tomographic coronary calcium scanning: a review and guidelines for use in asymptomatic persons. Mayo Clin Proc. 1999;74(3):243-252. doi:10.4065/74.3.243

19. Miyoshi M, Inoue Y, Nishioka M, et al. Clinical evaluation of presepsin considering renal function. PLoS One. 2019;14(9):e0215791. doi:10.1371/journal.pone.0215791 
20. Nakayama M, Ura Y, Nagata M, et al. Carotid artery calcification at the initiation of hemodialysis is a risk factor for cardiovascular events in patients with end-stage renal disease: a cohort study. $B M C$ Nephrol. 2011;12:56. doi:10.1186/1471-2369-12-56

21. Kobayashi S, Amano H, Terawaki H, Kawaguchi Y, Yokoo T. Prediction of presepsin concentrations through commensurate decline in kidney function in the elderly. Clin Chim Acta. 2020;500:1-9. doi:10.1016/j.cca.2019.09.012

22. Jofré R, Rodriguez-Benitez P, López-Gómez JM, Pérez-Garcia R. Inflammatory syndrome in patients on hemodialysis. J Am Soc Nephrol. 2006;17(12 Suppl 3):S274-S280. doi:10.1681/ASN.2006080926

23. Yoon JW, Gollapudi S, Pahl MV, Vaziri ND. Naive and central memory T-cell lymphopenia in end-stage renal disease. Kidney Int. 2006;70:371-376. doi:10.1038/sj.ki.5001550

24. Betjes MG, Huisman M, Weimar W, Litjens NH. Expansion of cytolytic CD4+CD28- T cells in end-stage renal disease. Kidney Int. 2008;74:760-767. doi:10.1038/ki.2008.301
25. Meijers RW, Litjens NH, de Wit EA, et al. Uremia causes premature ageing of the T cell compartment in end-stage renal disease patients. Immun Ageing. 2012;9:19. doi:10.1186/1742-4933-9-19

26. Angeletti A, Zappulo F, Donadei C, et al. Immunological effects of a single hemodialysis treatment. Medicina (Kaunas). 2020;56(2):71. doi:10.3390/medicina56020071

27. Block GA, Spiegel DM, Ehrlich J, et al. Effects of sevelamer and calcium on coronary artery calcification in patients new to hemodialysis. Kidney Int. 2005;68:1815-1824. doi:10.1111/j.15231755.2005.00600.x

28. Alesutan I, Luong TTD, Schelski N, et al. Circulating uromodulin inhibits vascular calcification by interfering with pro-inflammatory cytokine signaling. Cardiovasc Res. 2020:pii: cvaa081. doi:10.1093/ cvr/cvaa081.

29. Vervloet M, Cozzolino M. Vascular calcification in chronic kidney disease: different bricks in the wall? Kidney Int. 2017;91:808-817. doi:10.1016/j.kint.2016.09.024
Risk Management and Healthcare Policy

\section{Publish your work in this journal}

Risk Management and Healthcare Policy is an international, peerreviewed, open access journal focusing on all aspects of public health, policy, and preventative measures to promote good health and improve morbidity and mortality in the population. The journal welcomes submitted papers covering original research, basic science, clinical \& epidemiological studies, reviews and evaluations,
Dovepress

guidelines, expert opinion and commentary, case reports and extended reports. The manuscript management system is completely online and includes a very quick and fair peer-review system, which is all easy to use. Visit http://www.dovepress.com/testimonials.php to read real quotes from published authors. 